\title{
Chatter detection algorithm based on machine vision
}

\author{
Michał Szydłowski • Bartosz Powałka
}

Received: 21 April 2011 / Accepted: 28 November 2011 /Published online: 13 December 2011

(C) The Author(s) 2011. This article is published with open access at Springerlink.com

\begin{abstract}
Production of precise high-value mechanical elements requires a hundred percent on-site control. Chatter may occur due to random events. Although an unaided human eye can also easily identify the presence of chatter marks, it is economically ineffective. Therefore, an algorithm based on machine vision signals was proposed for surface inspection. The algorithm was designed to build an error map of the examined surface and highlight the regions of probable imperfections. The algorithm is based on local gradient estimation, applied in respect to milling parameters. Estimated local gradient directions are used to calculate the ridge/valley orientations of the machined surface. The local ridge orientation is used for the purpose of computing the surface error map. An experiment was made to test the algorithm. Milled surfaces in both chatter-free and chatterrich conditions have been analysed with the presented method.
\end{abstract}

Keywords Chatter detection · Machine vision · Image processing $\cdot$ Surface inspection

\section{Introduction}

Increased quality and efficiency requirements concerning elements of machines and devices cause an increase in the interest of manufacturing companies in autonomic control and monitoring systems. Therefore, it is justified to seek

\footnotetext{
M. Szydłowski $(\triangle) \cdot$ B. Powałka

Faculty of Mechanical Engineering and Mechatronics,

West Pomeranian University of Technology,

al. Piastów 17,

70-310 Szczecin, Poland

e-mail: michal.szydlowski@zut.edu.pl
}

methods which can automatically detect defective elements and send them to correction. One hundred percent control is applied in many branches of machine industry. Quality control systems and online measurement tools are commonly used for the supervision and control of production processes which result in the increase of their economic effectiveness. Chatter marks on the surface can be easily identified by the human eye, but in a hundred percent controlled production, it is impossible and economically unacceptable. CNC machine tools are equipped with increasingly accurate online monitoring systems. The literature shows many application examples of monitoring and control of machining processes [1]. The development of computer technology and the increase in the efficiency of microprocessor systems allowed for the application of new techniques. Therefore, solutions, methods and algorithms which allow for the assessment of products manufactured in the machining processes with the use of machine vision are currently being developed $[1,2]$. Solutions applied in the system of cutting edge wear monitoring are known from the literature. The aim of the methods applied there is to assess the degree of cutting edge wear on the basis of the tool geometry or texture analysis $[2,3]$. The method based on the examination of cutting edges by means of edge detection techniques in search of material defects [4] is also studied. Research centres conduct research within the scope of machine vision for the assessment of surface roughness. The applied techniques are based on neural network classifiers [5] or texture analyses [6,7]. Approaches encompassing the application of techniques based on the optical fibre technology [8] or connected with the use of infrared [9] are also known. There are a significant number of publications concerning the assessment of parameters of geometric surface structure on the basis of images [5, 8-10]. There are several known cases when surface defects generated as a 
result of machining remained unnoticed despite the measurement of the geometric structure of the surface [11]. Imperfections such as cracks caused by chips at many critical machine parts are unacceptable. Deviations of this nature include also traces formed as a result of machining in unstable conditions. Chatter in the tool-workpiece system causes many unwanted effects $[11,12]$. In production processes, a proper selection of machining parameters allows us to avoid many problems connected with the undesirable phenomenon of vibrations. Undesirable vibrations may occur due to the variability of production conditions, wear of machines, or e.g. incorrect fixturing of the machined item. Moreover, chatter causes the deterioration of the machine tool precision. The wear of the machine tool and its parts is accelerated significantly. It also causes the loss of geometric accuracy of the machined item. Therefore, the detection of machining defects in the production process is especially important. Not much place has been devoted to the research concerning the vision technique for the assessment and detection of chatter traces. Khalifa et al. [13] presented research concerning chatter traces on the surface images obtained in the machining. The authors used the gray-level co-occurrence matrix method; however, only two samples with extremely different surfaces were analysed. The edge detection method was also proposed [14]. This method is, however, sensitive to the illumination conditions (uniform illumination of the examined surface).

As results from the presented literature review, a large advantage would be the creation of a system for the assessment of surface quality. Vision systems give us an opportunity to make fast measurement and introduce a $100 \%$ control in a given production process. So far, there have been no attempts to use the machining parameters of the examined surface in the methods of image processing. Taking into account such values as the feed rate or the cutting speed may increase the efficiency of the analysis method. The aim of the presented research was to develop a method of image analysis which is dependent on the machining parameters. The article presents the kinematics of the milling process and the way of shaping the machined surface (Section 2). The machining parameters have a significant impact on the selection of parameters of the proposed algorithm. Surface defects occurring in the cases of machining in unstable conditions in the presence of chatter have also been presented (Section 2.1). A method for processing and analysis of images of the surface machined in the milling process which takes into consideration the machining parameters has also been presented (Section 3). The method was based on solutions applied in dactyloscopy. The experiment conducted in order to determine the efficiency of the method was described in detail (Section 4). In the article, the authors presented also the conclusions concerning the obtained results (Section 5).

\section{Milling process kinematics}

The kinematics of the milling process is in the simplest case a composition of two movements of the machine: the rotational motion and the translational motion. If a tool with a $z$ number of cutting edges rotates with a constant rotational speed of $N$ (revolutions per minute) and at the same time moves along a straight line with the velocity of $V_{\mathrm{f}}$ (metres per minute), then the point on the cutting edge distant by the value of the milling cutter diameter $r$ (millimetres) from the rotation axis of the tool has a cycloidal trajectory (Fig. 1). The location of the point on a cycloidal curve at a given time $t$ may be obtained from a simple dependence described by a system of Eq. 1. The absolute distance which the point of the cutting edge makes during one rotation of the tool is called feed per revolution of the milling cutter $f_{\mathrm{r}}$ (millimetres per rotation] (Eq. 2).

$\left\{\begin{array}{c}x(t)=V_{\mathrm{f}} t+r \cos (2 \pi N t) \\ y(t)=r \sin (2 \pi N t)\end{array}\right.$

$f_{\mathrm{r}}=\frac{V_{\mathrm{f}}}{1,000 \cdot N}$

In the case of a run with the whole diameter of the mill, the cutting edges of the tool work in the material

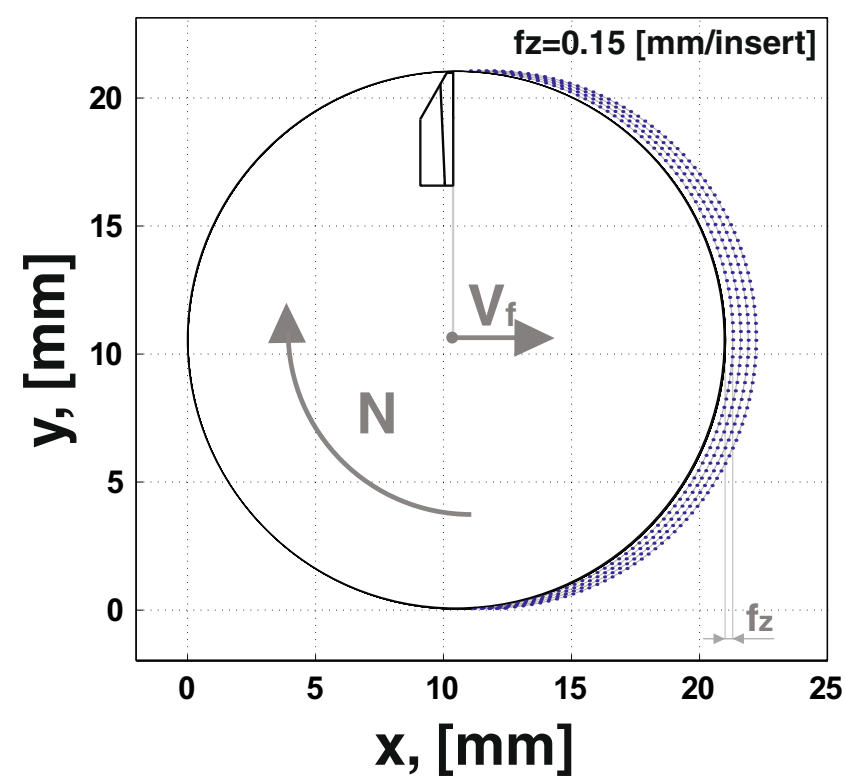

Fig. 1 Generated trajectory of a point situated on the cutting edge for one insert 
only within the angular range from $0^{\circ}$ to $180^{\circ}$. From the trajectory obtained on the basis of Eq. 1, we should exclude angular ranges where insert are not cutting. The exclusion should provide points of traces made by the cutting edge on the machined surface. Knowing the machining parameters and the diameter of the tool, we are able to determine the places where there are ridges on the machined surface.

Analysing the image of the milled surface presented in Fig. 2, we are able to see brighter and darker areas of pixels placed alternately presenting traces of machining. The image profile gives us more information and allows us to determine that the brighter areas are situated higher than the darker ones.

Therefore, traces left by the edges of the milling cutter form ridges and valleys. The distance between the adjacent ridges is equal to $f_{z}$ :

$f_{z}=\frac{f_{\mathrm{r}}}{z}$

\subsection{Surface defects}

Surfaces shaped during the milling process in the conditions of occurrence of undesirable vibrations have certain features which allow an experienced quality controller to assess their intensity. Vibrations in the system of the tool and the workpiece cause the occurrence of characteristic traces. Vibrations cause displacement of the tool, due to which the trajectory of the point located on the cutting edge changes (Fig. 3).

Surfaces performed with chatter have a variable width of valleys and ridges left by the cutting edges (Fig. 4). In the case of the last surfaces (Fig. $4 \mathrm{~d}, \mathrm{e}, \mathrm{f}$ ), it can be said

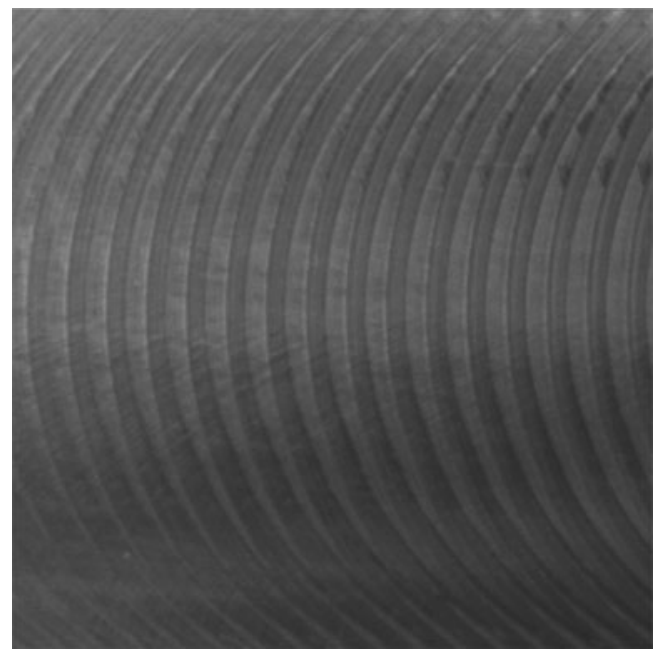

Fig. 2 Image of a fragment of surface after milling in stable conditions; traces of the tool create a system of ridges and valleys

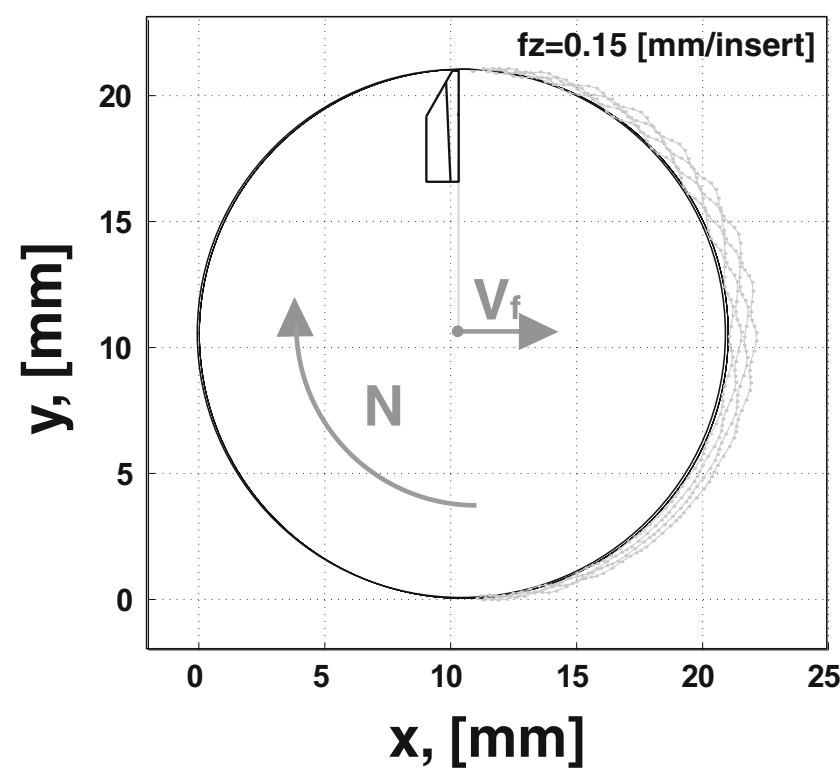

Fig. 3 Trajectory of a point situated on a cutting edge - vibrations in the machining process generated by an addition of an error in the orientations of $x$ and $y$, expressed by a sinus function with the amplitude of $1 / 2 f_{z}$

that there is a downright discontinuity of those traces. It is also clearly visible that the periodical character of the texture created by the surface has changed. It is also hard to determine unequivocally the parts of cycloidal curves formed as a result of machining-the system of ridges and valleys is thus disturbed. Therefore, we can expect that the orientations of the image's local gradients will be arranged in a different way than in the case of images of surfaces milled in chatter-free conditions.

It can be assumed that the orientation of ridge lines within the image will be variable. This fact served for the preparation of the analysis method of the surface after milling described below. Milled surfaces performed in non-chatter conditions display high periodicity, and any deviations may be a signal of surface defects such as vibrations which occur during machining, cracks and breaks.

\section{Algorithm of surface defect detection}

Heterogeneous character of traces of milling cutter cutting edges on the machined surface with surface chatter is possible to discern for an unarmed human eye. Having the information concerning the machining parameters and using the fact that on the defective surfaces there is a heterogeneity of shape and location of cutting edge traces, a method based on the analysis of the local gradient orientations was proposed. The presented method was inspired by the 
Fig. 4 Surfaces performed in the case of different machining depths $a_{\mathrm{p}} ; \mathbf{a}, \mathbf{b}, \mathbf{c}$ stable conditions; $\mathbf{d}$, e unstable conditions; $\mathbf{f}$ unstable conditions (the highest value $a_{\mathrm{p}}$ )
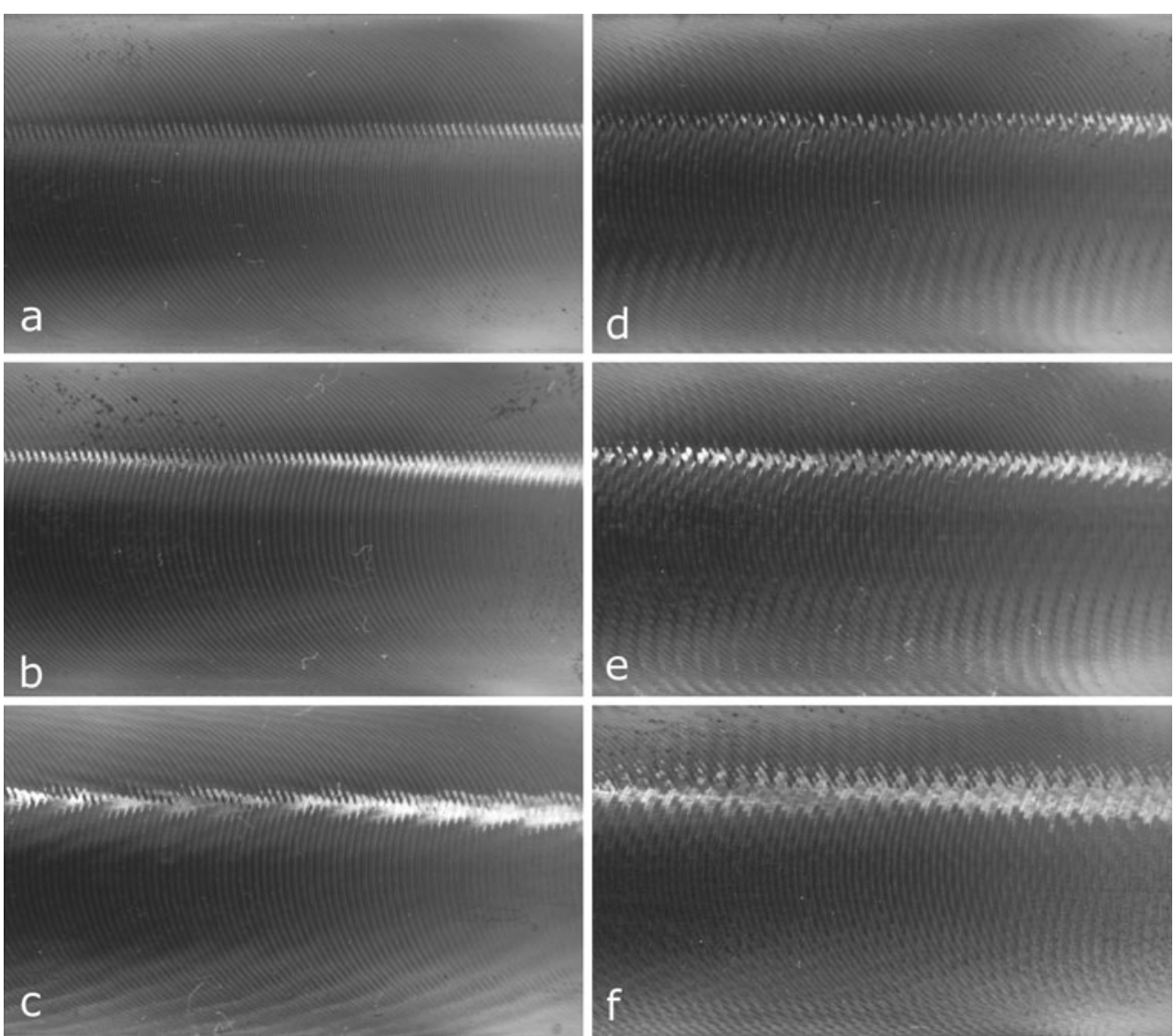

achievements in the area of biometric analysis, in which it is used for fast recognition and comparison of characteristic human fingerprint [15]. The method consists of four stages. The first stage is the image normalisation which allows for the comparison of surface images taken in different illumination conditions. The second stage involves conducting a division of the image into blocks which allows for determining the areas in which the ridge orientations will be assessed. The third step is a selection of proper gradient operators which allow for the assessment of ridge orientations. The last stage of this method is based on finding defects on the surface by means of determining local variances of ridge line orientations. The result of the last stage is a map of surface defects.

\subsection{Image normalisation}

The aim of the normalisation is to bring an unknown distribution of pixel illumination value of the image to the distribution of known parameters of the average and the variance, which will allow us to obtain uniform results for the group of images (other illumination conditions). According to the definition proposed by Gonzalez [16], an image $f=I(i, j)$ is a function where, for each pair of coordinates $i, j$, an intensity value $f$ of an elementary image fragment is assigned. For a digital image, the sets $i, j$ and $f$ have finite value. Intensity value $f$ of pixels is usually expressed by the number of bits, e.g. for eight bits, we assume the value from 0 to 255 . For a digital image represented by the matrix $I$ of size $X \times Y$, with variance $s^{2}$ and the mean $\bar{x}$, and an assumed variance $s_{n}{ }^{2}$ and mean $\bar{x}_{n}$ of the new target distribution, the normalised image of $M(i, j)$ is given by expression 4 . It should be noted that the estimators $\bar{x}$ and $s^{2}$ are defined by expressions 5 and 6 , respectively.

$M(i, j)=\bar{x}_{n}+\frac{s_{n}^{2}(I(i, j)-\bar{x})^{2}}{s^{2}}$

$\bar{x}=\frac{1}{X \cdot Y} \sum_{i=1}^{x} \sum_{j=1}^{y} I(i, j)$

$s^{2}=\frac{1}{X \cdot Y} \sum_{i=1}^{x} \sum_{j=1}^{y}[I(i, j)-\bar{x}]^{2}$

\subsection{Image division}

In order to determine the lines of ridges, the image needs to be divided into processing blocks in which the local 
assessment of gradient orientations will take place. The size of these blocks should be selected in such a way to fit one valley and one ridge. If we assume that the image has $X \times Y$ pixels, it should be divided into equal blocks $W_{m}(i, j)$ where the pair of coordinates $(i, j)$ is the centre of the $m$ square block of pixels with side $l$. If the division of the image into equal blocks is impossible, the image should be framed properly so that the $X$ dimension and the $Y$ dimension of the image were evenly divisible by $l$. Since we know the pixel dimension, we are able to determine the length of the $l$ side corresponding to the feed per tooth $f_{z}$. It should also be remembered that the blocks have to consist of an odd number of pixels [17].

\subsection{Gradient orientation}

The ridge orientation in the image $I$ may be indicated by means of the gradient method [18]. For a block of the image $W(i, j)$ with a central element with the coordinates of $(i, j)$, the local gradient orientation $\Theta(i, j)$ will be normal to the orientation of the ridge or valley at the same time. It is impossible to determine the sense of the gradient in such a method. It should be noted that e.g. the values of $\Theta 90^{\circ}$ and $270^{\circ}$ shall indicate the same orientation. For determining orientation $\Theta$, it is necessary to calculate gradient values for each block $W(i, j)$ for two orientations, i.e. $\partial_{x}(i, j)$ and $\partial_{y}(i, j)$. One of the known operators, such as Sobel, Scharr and Marr-Hildret may be applied for calculating gradients. Within the framework of this method, an operator matching the resolution of the image and the geometry of ridges and valleys was determined. Assuming that $H$ is the image of the Gaussian filter $H(\sigma, h)$ with a standard deviation $\sigma$ and the window length $h$, we can calculate the gradient value and the $H$ filter image value and use them as operators for determining image $I$. The filter parameters should be selected properly to the size of the surface images so that the window length was similar to the size of the ridge (traces of the milling cutter cutting edge-i.e. $h=$ $f_{z}$ ). Another stage is the assessment of ridge orientation. To this end, Hong, Wan and Jain [18] proposed a method of the least squares. Ridge orientations $\Theta(i, j)$ for each block (Eq. 9) are determined by means of covariance coefficient Eqs. 7, 8,

$V_{x}(i, j)=\sum_{u=1}^{l} \sum_{v=1}^{l} 2 \partial_{x}\left(u+i-\frac{l-1}{2}, v+j-\frac{l-1}{2}\right) \partial_{y}\left(u+i-\frac{l-1}{2}, v+j-\frac{l-1}{2}\right)$

$V_{y}(i, j)=\sum_{u=1}^{l} \sum_{v=1}^{l} 2\left[\partial_{x}\left(u+i-\frac{l-1}{2}, v+j-\frac{l-1}{2}\right)^{2}-\partial_{y}\left(u+i-\frac{l-1}{2}, v+j-\frac{l-1^{2}}{2}\right)\right]$

$\Theta(i, j)=\frac{1}{2} \tan ^{-1}\left(\frac{V_{y}(i, j)}{V_{x}(i, j)}\right)$

Hong et al. state that mathematically, $\Theta(i, j)$ represents the direction that is orthogonal to the dominant direction of the Fourier spectrum of the $W(i, j)$ window. Due to the noise present in the image, weak resolution and other factors, such an assessment process may be inaccurate. Therefore, a lowpass filter $F(2 l, h)$ was used to determine $\Theta(i, j)$ in a more precise way. The low-pass filter selection is justified assuming that frequencies connected with the ridges and valleys are the lowest frequencies of the image. In order to conduct filtration, it is necessary to convert matrix $\Theta$ into a continuous vector field:

$\Phi_{x}(i, j)=\cos (2 \Theta(i, j))$

$\Phi_{y}(i, j)=\sin (2 \Theta(i, j))$
Then, smoothing should be conducted by means of twodimensional filter $F$ of the window length of $h_{F}$ according to the following:

$\Phi_{x}^{\prime}(i, j)=\sum_{u=1}^{h_{f}} \sum_{v=1}^{h_{f}} F(u, v) \Phi_{x}\left(i+u+\frac{h_{f}-1}{2}, j+v+\frac{h_{f}-1}{2}\right)$

$\Phi_{y}^{\prime}(i, j)=\sum_{u=1}^{h_{f}} \sum_{v=1}^{h_{f}} F(u, v) \Phi_{y}\left(i+u+\frac{h_{f}-1}{2}, j+v+\frac{h_{f}-1}{2}\right)$

On the basis of data prepared in such a way, it is possible to determine the ridge orientations $\Theta^{\prime}$ :

$\Theta^{\prime}(i, j)=\frac{1}{2} \tan \left(\frac{\Theta_{y}^{\prime}(i, j)}{\Theta_{x}^{\prime}(i, j)}\right)$ 


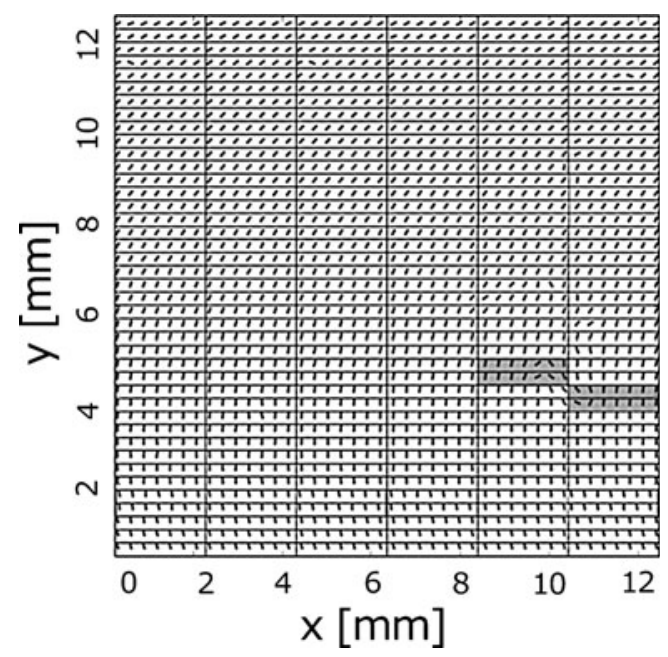

Fig. 5 Visualisation of an exemplary matrix division. Dark areas are characterised by high variability of ridge orientation

\subsection{Map of surface defects}

Ridge line orientations are locally characterised by low variability in the case of surfaces performed in the process in which chatter was not present. If we separate rows from the matrix and divide each one into subsets with the same number of elements-we shall obtain a set of orientations corresponding to concrete areas of the surface image. For a surface performed without the presence of chatter, the determined ridge line orientations should be characterised by low variability. Deter- mining the orientation variance of local ridges within the separated areas, we are able to build a map of orientation change. If the orientations are characterised by high variability within any area (high variance value), it means that a surface defect may occur within these areas. The division method has been presented in Fig. 5. Taking all the variance levels for one surface, an average variance level parameter was calculated. The parameter was used besides the error map to compare the examined surfaces.

\section{Research verification of methods}

Research station presented in Fig. 6 consisted of a CCD Pulnix recorder mounted on a stand. Two illuminators were applied in order to illuminate the surface of metal samples equally. The CCD camera was connected to a PC which was responsible for the image acquisition. The image analysis was conducted by means of the MATLAB software along with the Image Processing Toolbox package.

Seven surfaces were prepared as a result of milling grooves. Forces and accelerations for all operations were registered during milling by means of a measurement system equipped with a Kistler dynamometer (model 9443B) and an accelerometer (mounted on a spindle). Data were recorded so that it could be possible to state whether chatter was present during machining and to determine their inten-
Fig. 6 Setup used for image acquisition

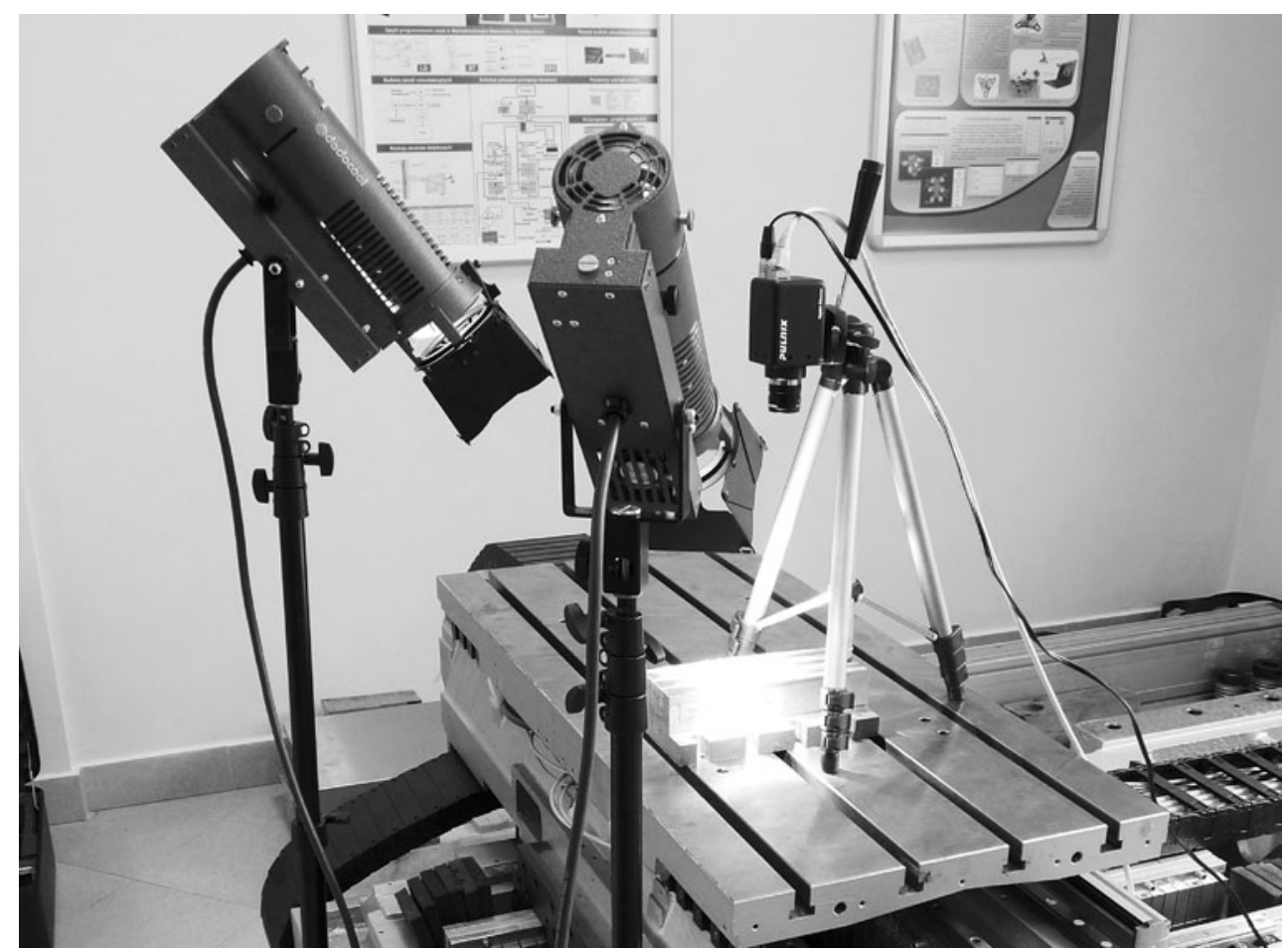



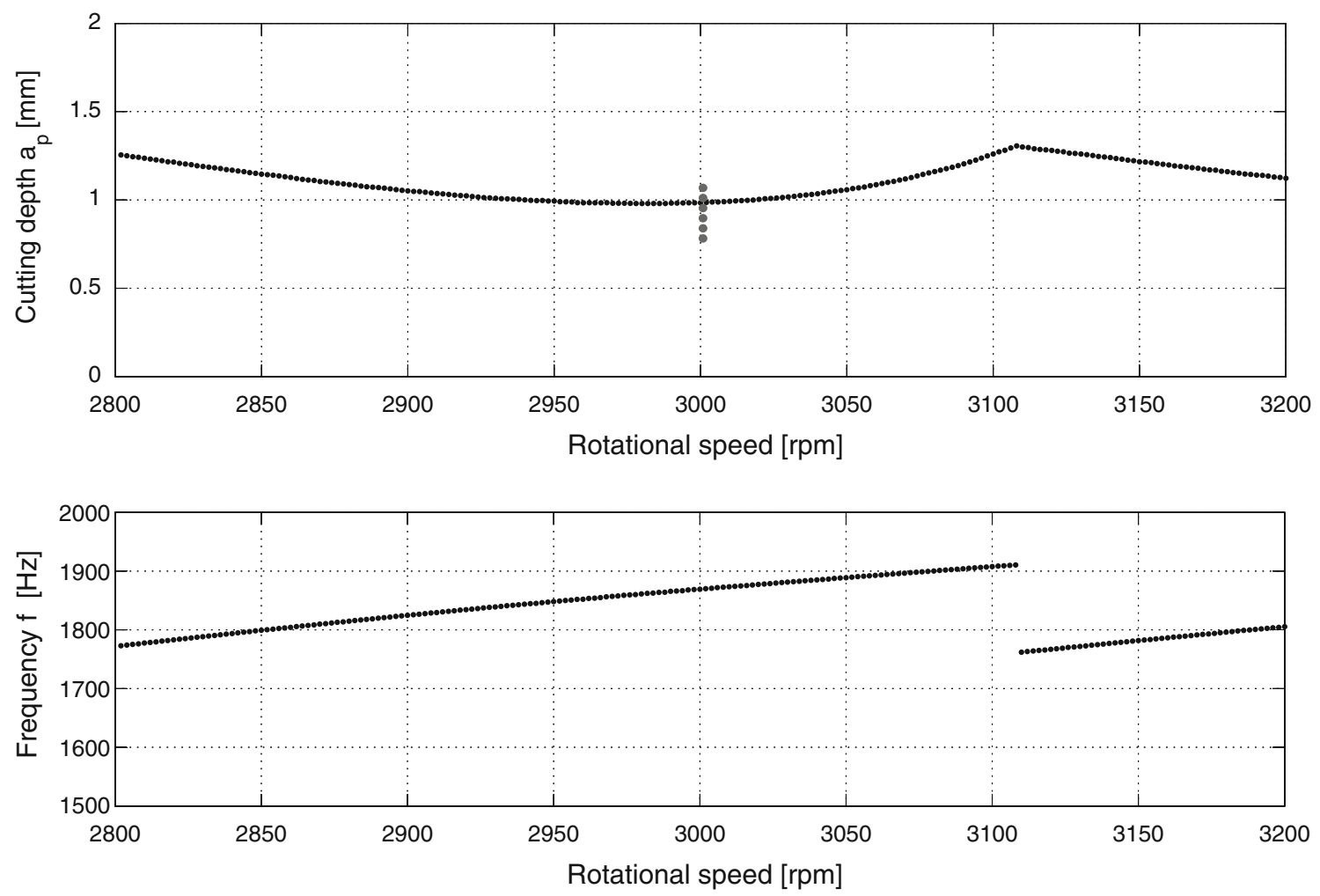

Fig. 7 Stability lobes diagram which allows for determining the areas of unstable machining

sity. Machining parameters were selected on the basis of stability lobes diagrams [12]. The obtained stability lobes diagrams have been presented in Fig. 7. The area located below the curve determines the work in stable conditions. The area above the curve is the area of chatter occurrence. Stability lobes diagrams allow us to determine the depth of machining in the case of which the occurrence of chatter is expected for a fixed rotational speed of the machine tool spindle. The research was made on DMG DMU 40 machine, using ISCAR HM90 E90A-D21-3-C20 (diameter, $21 \mathrm{~mm}$ ) mill with three HM90 APKT 1003PDR IC908 inserts. Surfaces were performed with the constant feed rate $(0.15 \mathrm{~mm})$ and rotational speed $(3,030$ rotations $/ \mathrm{min})$. Whereas for further grooves, the cutting depth was changed within the scope from 0.8 to $1.1 \mathrm{~mm}$ every $0.05 \mathrm{~mm}$. Pictures of the samples were taken with the resolution of $1,100 \times 900$ pixels, with unchanged conditions of illumination and recorder settings. Due to the fact that the optics did not allow for taking a picture of the performed groove surface, the images were cropped to the resolution of $1,000 \times 500$. In the algorithm, the length of the filter window $\mathrm{H}$ was determined at the level of $l=7$ pixels (the $f_{z}$ value translated into pixels), whereas $\sigma=1$. Gaussian filter $\mathrm{F}$ with the parameters of $\sigma=3$ and $h=15$ was used for smoothing. The value of the $h$ parameter of filter $\mathrm{F}$ is equal to the double of $f_{z}+1$, since at least two ridges in the widow are necessary for filtration and the window itself needs to be odd. And therefore, the sizes of the $\mathrm{H}$ and $\mathrm{F}$ filter windows were determined in a way to correspond to the value of parameters applied in the case of machining, i.e. feed per tooth. Additional measurements were made with HOMEL T1000 Surface Analyzer. Contact profilometry was used to check if there is any correlation between the surface parameters such as $r_{a}$ with chatter mark presence.

\section{Results of the experiment}

It was possible to obtain images with marked orientations of the ridge line. Figure 8 presents two surface samples.

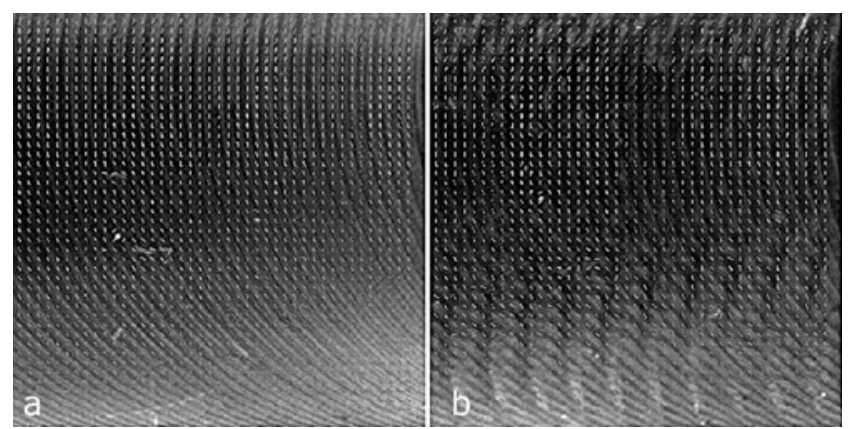

Fig. 8 Images of test surfaces with the ridge line orientations 


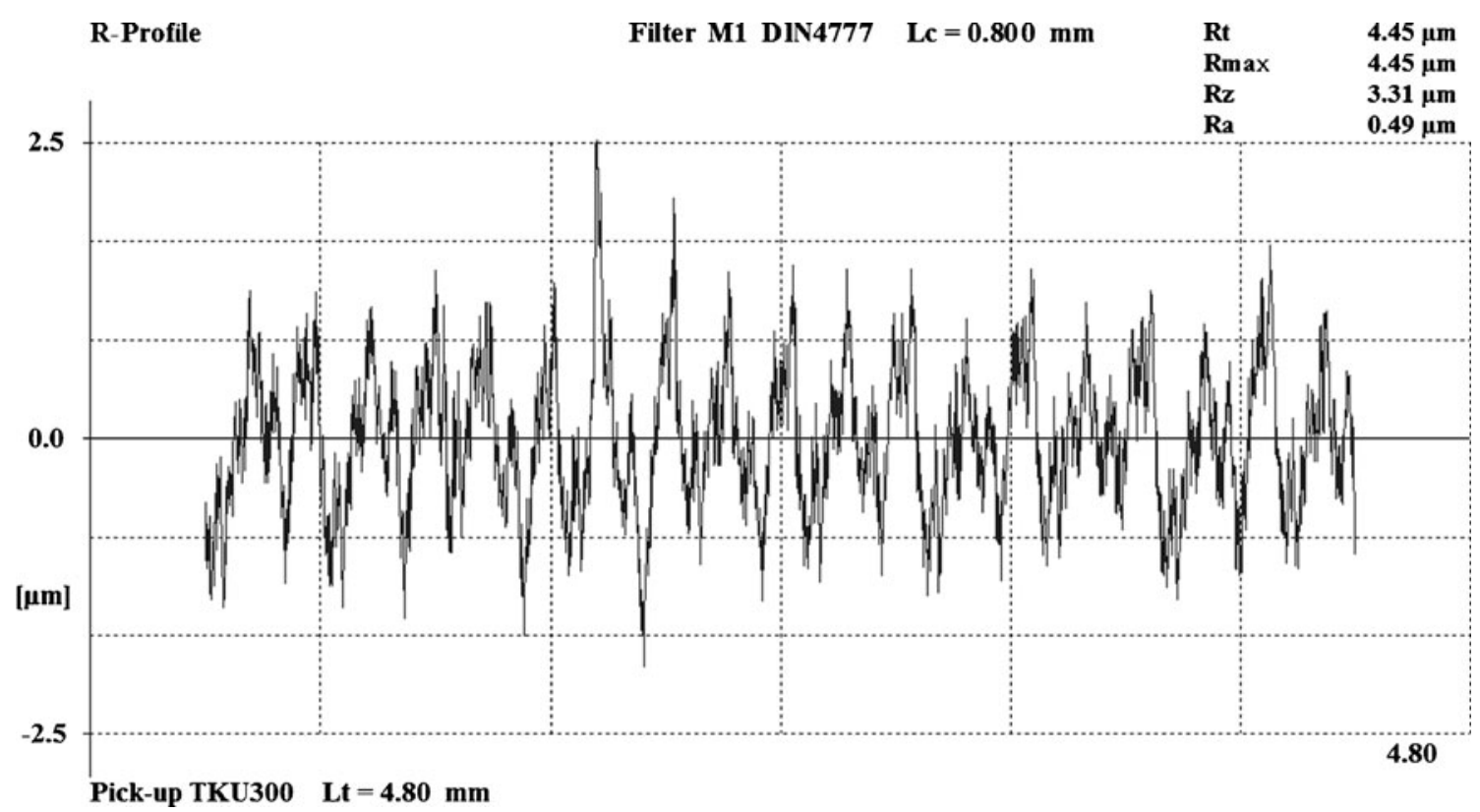

Fig. 9 The profile and key parameters of a chatter-free surface

Figure 8a presents the surface performed in stable conditions. All surfaces performed for the value had similar parameters. The presented figure shows how the assessed orientations of ridge lines overlap with the actual traces of the tool work. In the place where cracks are visible on the sample, the deviation of the assessed orientation takes place. It is similar in the cases where there are breaks, greasiness and foreign matter (e.g. fibre fragments) on the surface. In the case of such defects, deviations from the traces left by the cutting edges occur locally in the area of the defect.

In the case of Fig. 8b, the surface was made in unstable conditions. The impact of chatter on the shaped surface is clearly visible. Deviations of ridge orientations from the expected trajectory of edges in the case of milling are significant and increase along with the increase of chatter in the tool-workpiece system. Places in which the deviations from the expected orientations

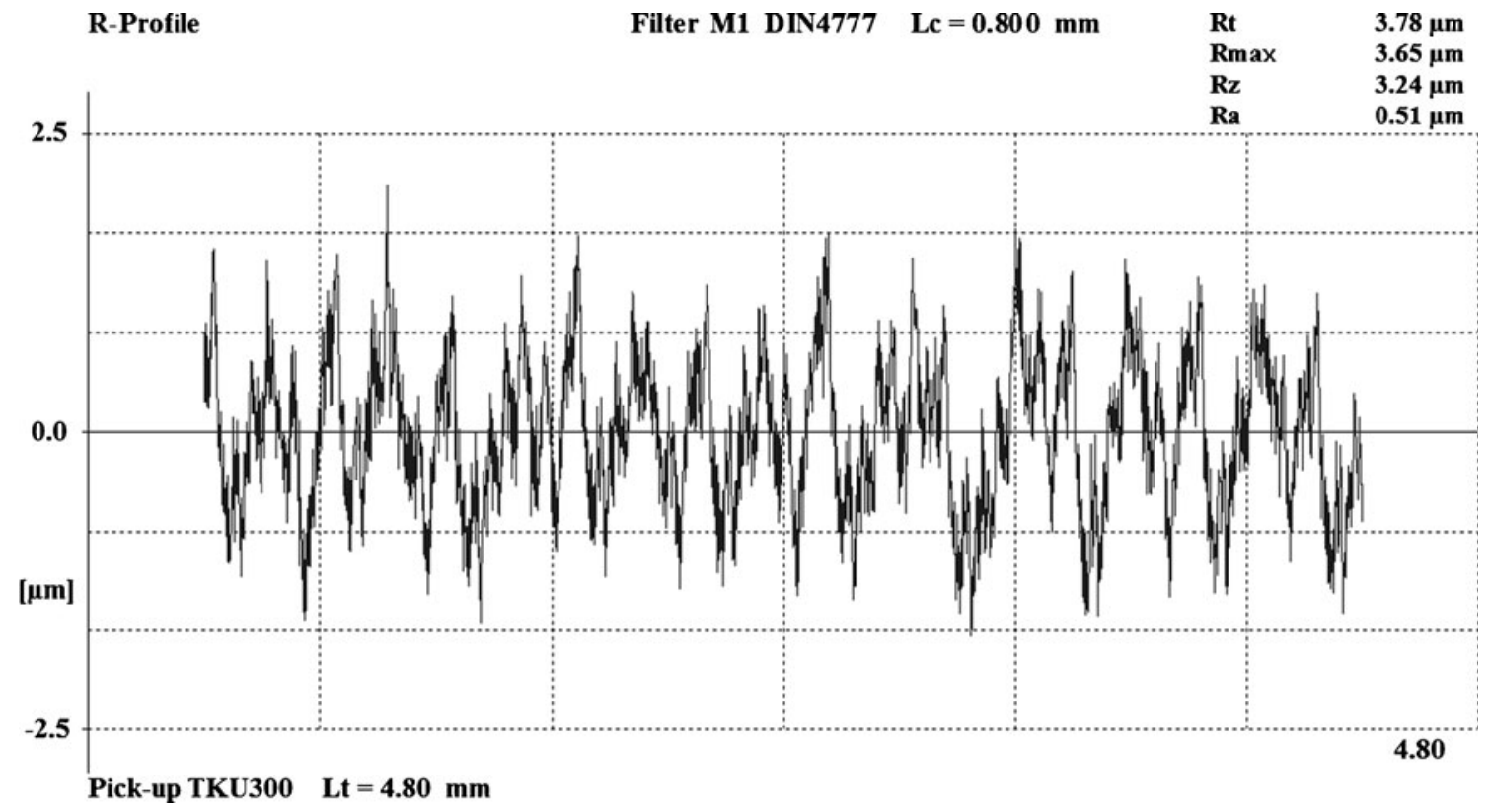

Fig. 10 Profile and key parameters of a chatter-rich surface 

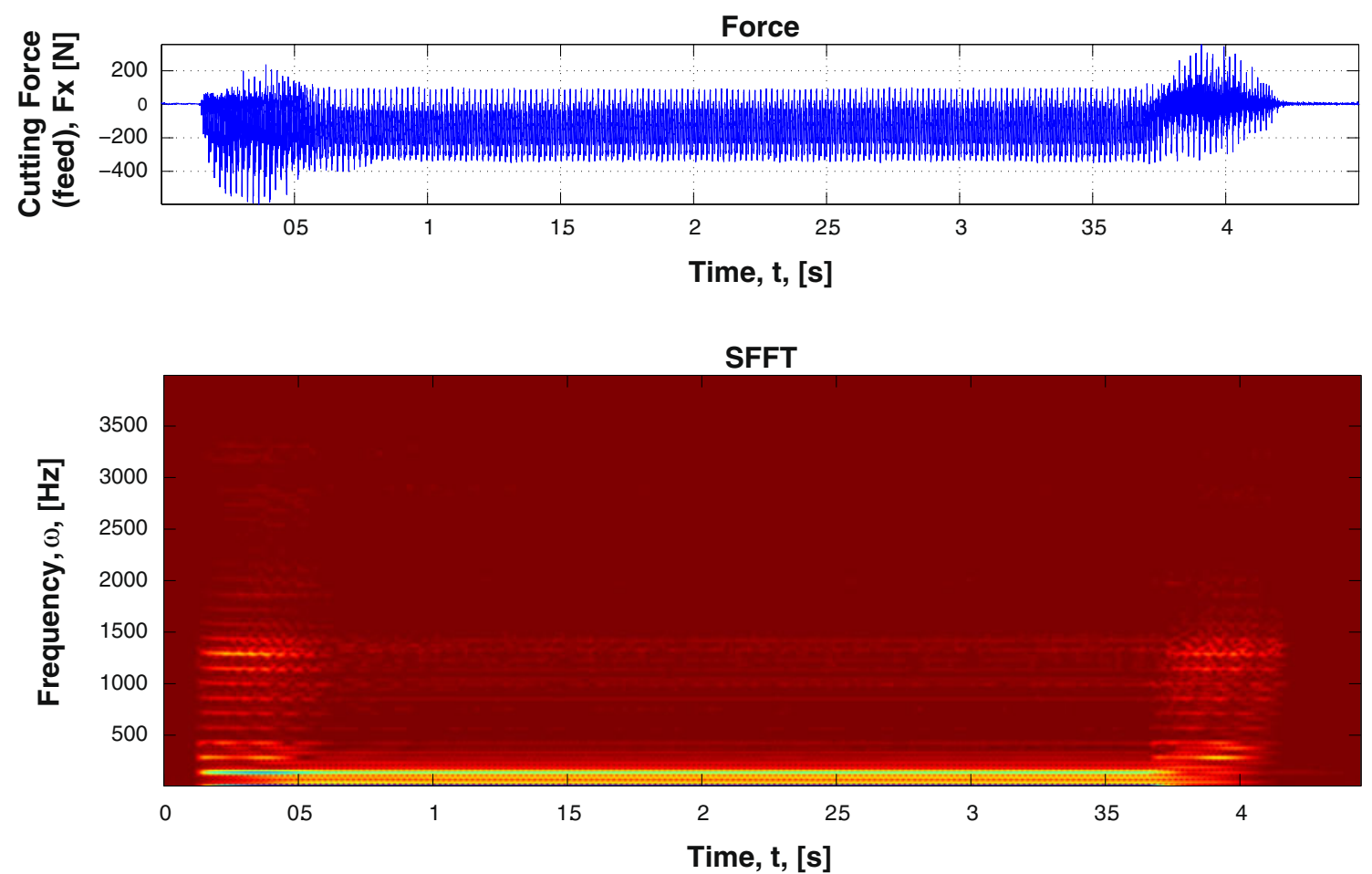

Fig. 11 Cutting force in feed direction with SFFT (chatter-free machining)

occur may suggest the occurrence of surface defects or greasiness.

The surfaces have been measured using contact profilometry. A typical group of parameters $\left(R_{\mathrm{a}}, R_{\mathrm{z}}, R_{\max }\right)$ was calculated with the results. The parameters for different groves did not differ in a significant way. Figures 9 and 10 present the key surface parameters with the filtered profile of a chatter-rich and chatter-
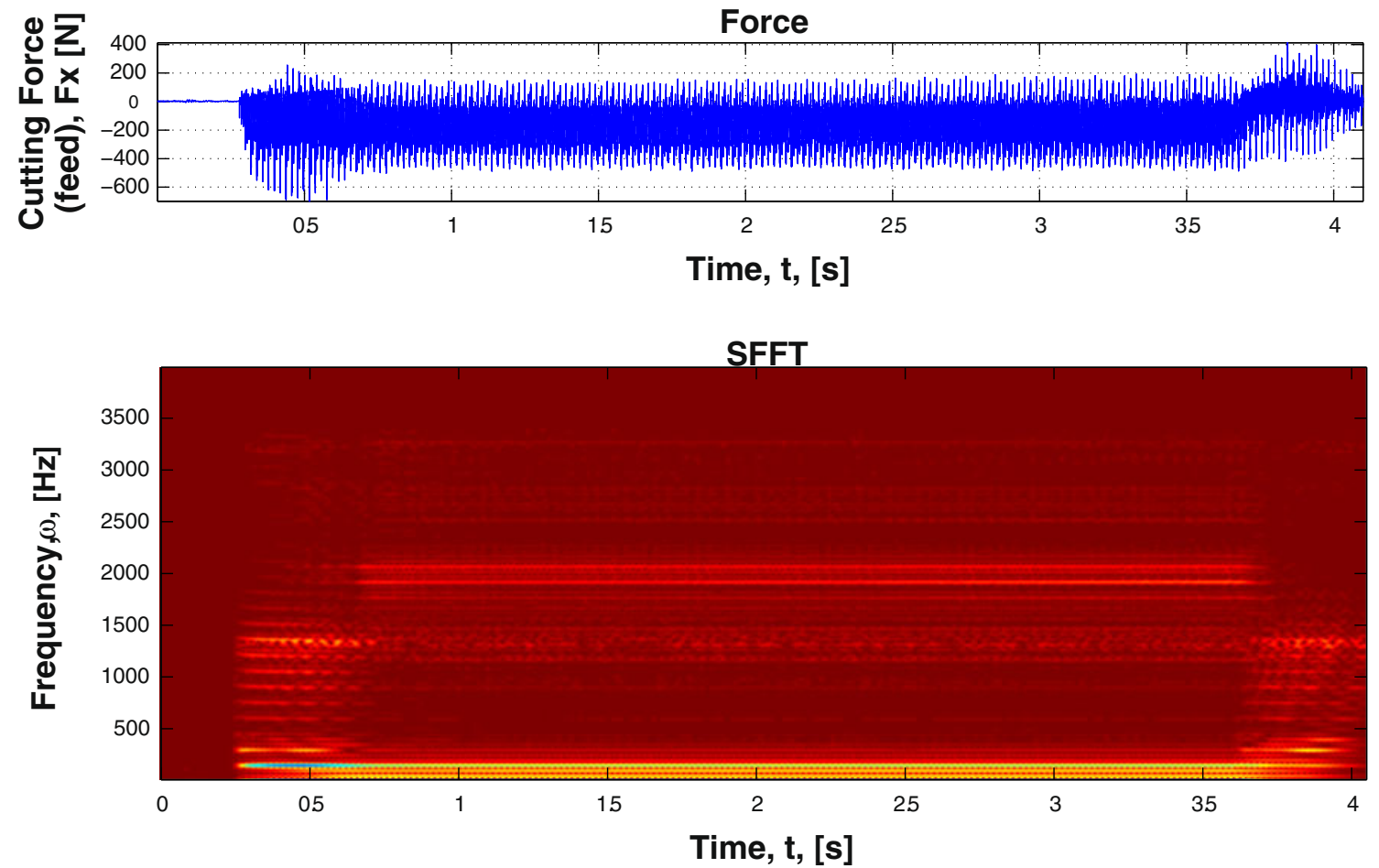

Fig. 12 Cutting force in feed direction with SFFT (chatter-rich machining) 
free surface. The profile was levelled and filtered using M1 DIN4777.

Figures 11 and 12 present force signals measured with the dynamometer and the Sparse Fast Fourier Transform (SFFT) of the signals. To determine if chatter was present during the machining, the SFFT was analysed for chatter frequency presence. Figure 11 presents cutting force measured in feed direction while machining. The figure shows that there is no chatter frequency present. On the other hand, Fig. 12 shows a frequency around $2 \mathrm{kHz}$ that can be associated with chatter. Surfaces described as chatter rich after the researchers' eye examination all had the $2-\mathrm{kHz}$ frequency present.

In order to present differences between surfaces where no chatter occurred during machining and surfaces in the case of which the results of chatter are visible, maps of orientation variability of ridge lines have been prepared (Figs. 13 and 14). The map obtained during the analysis has been presented in Fig. 10. The diagram presents the variability of individual image parts and allows for its comparison. A similar map has been prepared for the surface performed in stable conditions (Fig. 14). It should be noted that the average level of orientation variability expressed by the variance is lower than on the surface in the map presented in Fig. 13. The average variance levels were compared with the SFFT data from the machining. All surfaces which had the average variance level higher than 0.71 also had the $2-\mathrm{kHz}$ chatter frequency present in the force signal. Therefore, a threshold was set to 0.71 determining chatter presence in the surface image.

The average time of calculations conducted by the algorithm implemented in the MATLAB environment amounted to approximately $2.5 \mathrm{~s}$. The time measurement included uploading the image to the memory, conducting normalisation and the necessary calculations.
Fig. 13 Map of orientation variance of ridge lines for surfaces machined in unstable conditions; average variance level, 0.74
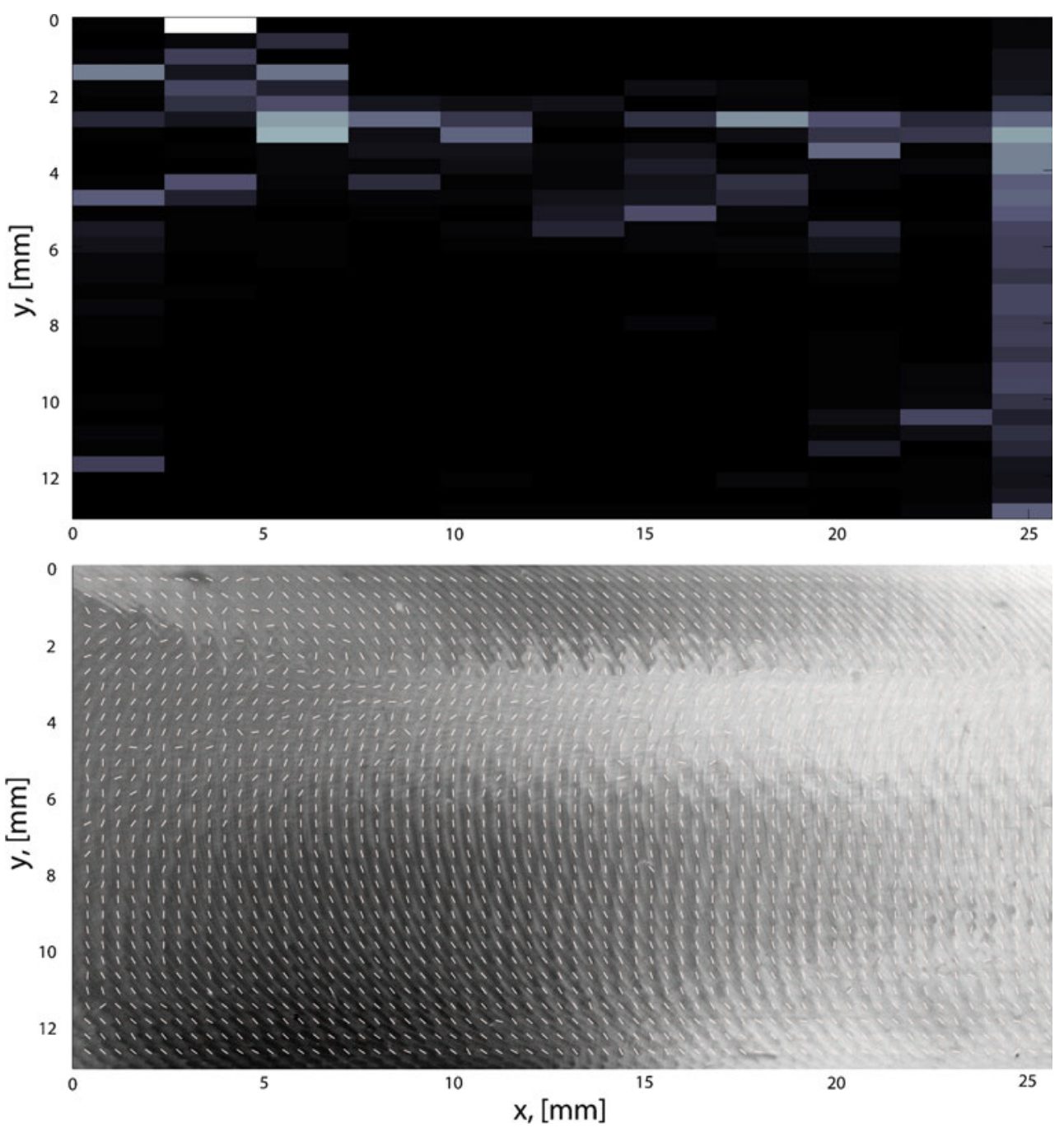
Fig. 14 Map of orientation variance of ridge lines for surfaces machined in stable conditions; average variance level, 0.59
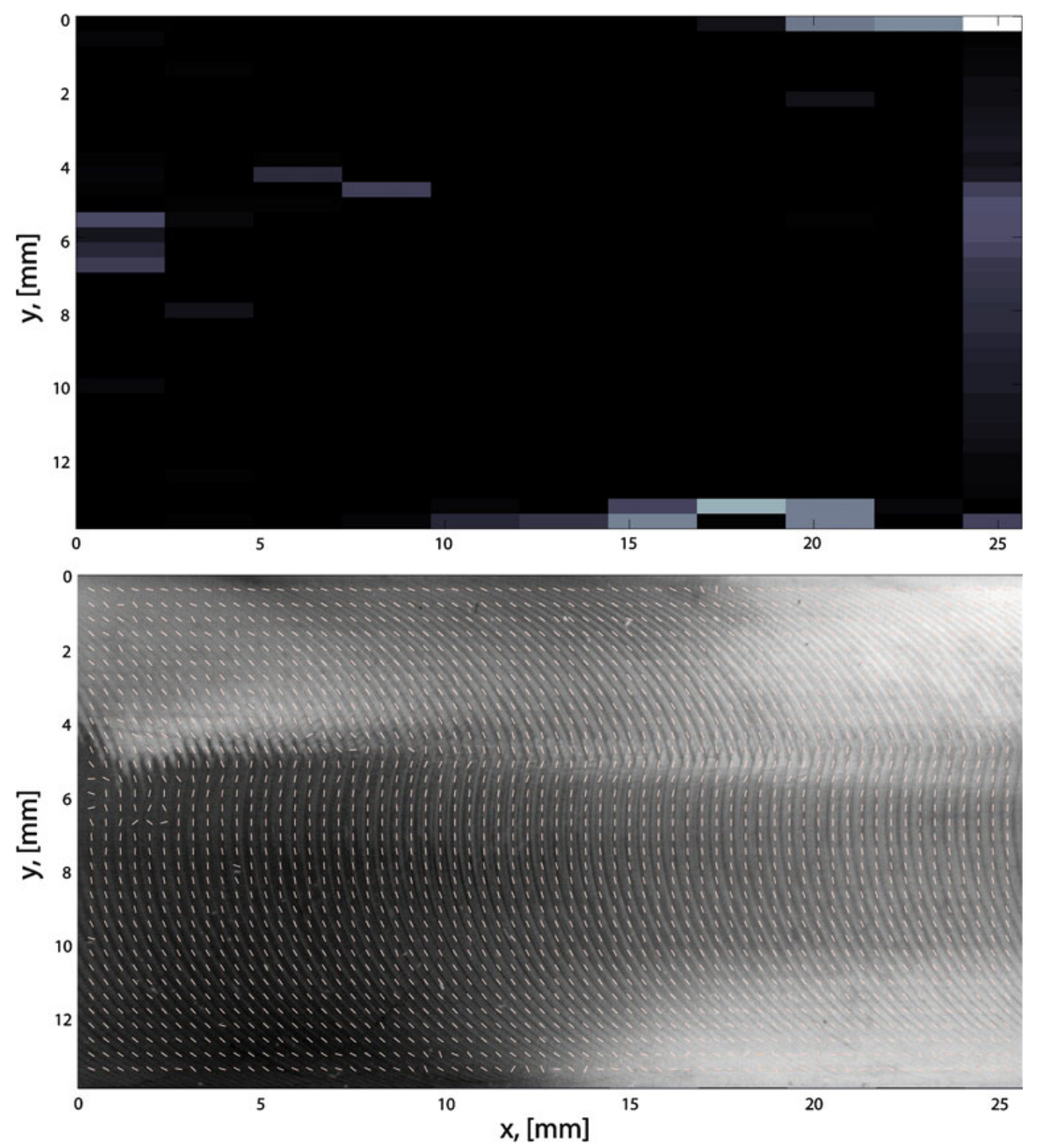

\section{Conclusion}

A part of the surface was machined in conditions in which chatter occurred. Comparison of images with marked ridge orientations leads to a conclusion that this method can be effective for the detection of surface defects caused by unstable machining conditions. The knowledge of machining parameters allows for a proper selection of the filter parameters and for the comparison of the assessed ridge orientations with the theoretical ones resulting from the kinematics of the process. This method may also indicate the presence of other surface defects, such as breaks, corrosion, cracks or greasiness. Obviously, if the nature of the defect is such that it will be overlapping with the orientations of the ridge lines, the method may occur to be ineffective. Such a situation may also take place for example in the case of cracks running in parallel to the traces from machining. An essential factor is also the resolution of images. It is important that the dimension of image pixels is smaller than the $f_{z}$ value. The analysis is feasible in the case of the $f_{z}$ value of approximately 7 8 pixels. As shown by the conducted experiments, framed fragments of such images may be applied interchangeably. In order not to omit significant features of the surface, it is possible to determine a few areas of interest which should present the image of the whole surface. The drawback of the approach presented in the article is that the decision criteria are threshold based and need calibration (threshold setting) for each process. To recapitulate, methods of image processing may become a potential control tool of milled surfaces. The algorithm proposed by the authors may become an effective control tool of the machining process owing to the application of processing parameters. 
Open Access This article is distributed under the terms of the Creative Commons Attribution Noncommercial License which permits any noncommercial use, distribution, and reproduction in any medium, provided the original author(s) and source are credited.

\section{References}

1. Liang SY, Hecker RL, Landers RG (2004) Machining process monitoring and control: the state-of-the-art. J Manuf Sci Eng 126

2. Cho D, Lee SJ, Chu CN (1999) The state of machining process monitoring research in Korea. Int J Mach Tools Manuf 30:16971715, Maryland Heights

3. Kassim AA, Mannan MA, Mian Z (2007) Texture analysis for tool condition monitoring. Image Vis Comput 25:1080-1090

4. Oguamanam DCD, Raafat H, Taboun SM (1994) A machine vision system for wear monitoring and breakage detection of single-point cutting tools. Comput Ind Eng 26(3):575-598

5. Al-Kindia G, Shirinzadehb B (2005) An evaluation of surface roughness parameters measurement using vision-based data. Int $\mathrm{J}$ Mach Tool Manuf 47:697-708

6. Zhenga H, Kongb LX, Nahavandia S (2007) Automatic inspection of metallic surface defects using genetic algorithms. J Mater Process Technol 125-126:427-433, Maryland Heights

7. Lee BY, Tarng YS (2007) Surface roughness inspection by computer vision in turning operations. Int $\mathrm{J}$ Mach Tool Manuf 41:1251-1263, Maryland Heights

8. Bradley G, Bohlmann J, Kurada S (1998) A fiber optic sensor for surface roughness measurement. J Manuf Sci Eng 120

9. Bjuggren M, Krummenacher L, Mattson L (1997) Non-contact surface roughness measurement of engineering surfaces by total integrated infrared scattering. Precis Eng 20:33-45
10. Kumar R, Kulashekar P, Dhanasekar B, Ramamoorthy B (2005) Application of digital image magnification for surface roughness evaluation using machine vision. Int J Mach Tool Manuf 45:228-234

11. Nurul Amin AKM, Anayet Patwari U, Sharulhazrin MS, and Hafizuddin I (2010) Investigation of effect of chatter amplitude on surface roughness during end milling of medium carbon steel. Proceedings of the 2010 International Conference on Industrial Engineering and Operations Management, Dhaka, Bangladesh, January $9-10$

12. Tomków J (1998) Wibrostabilność obrabiarek, Komputerowe wspomaganie obliczeń i badań doświadczalnych [Machine tools stability. Computer aided analysis and experimental investigations]. Wydawnictwo Naukowo-Techniczne, Fundacja Książka Naukowo Techniczna, Warsaw

13. Khalifa OO, Densibali A, Faris W (2006) Image processing for chatter identification in machining processes. Int $\mathrm{J}$ Adv Manuf Technol 31:443-449

14. Szydłowski M, Powałka B, Marchelek K (2010) Digital image processing in surface quality inspection. Proceedings of the ASME 2010 10th Biennial Conference on Engineering Systems Design and Analysis ESDA 2010 July 12-14, Istanbul, Turkey

15. Jain AK, Pankanti S (2000) Fingerprint classification and recognition. In: Bovik A (ed) The image and video processing handbook. Academic, New York, April 2000

16. Gonzalez RC, Woods RE, Eddins SL (2009) Digital image processing using MATLAB, 2nd edn. Gatesmark Publishing

17. Hong L, Wan Y, Jain AK (1998) Fingerprint image enhancement: algorithms and performance evaluation. IEEE Trans PAMI 20 (8):777-789

18. Hong L, Wan Y and Jain AK (1998) Fingerprint image enhancement: algorithms and performance evaluation. Proc. IEEE Comp. Soc. Workshop on Empirical Evaluation Techniques in Computer Vision, Santa Barbara. June 1998, pp. 117-134 